Check for updates

Cite this: RSC Adv., 2017, 7, 22415

\title{
Efficient photocatalytic dye degradation over Er-doped BiOBr hollow microspheres wrapped with graphene nanosheets: enhanced solar energy harvesting and charge separation
}

\author{
Chuang Liu, Xiaoli Dong, (D) * Yuchen Hao, Xiuying Wang, Hongchao Ma \\ and Xiufang Zhang
}

In this work, Er-doped BiOBr hollow microspheres wrapped with graphene nanosheets, which acted as a photocatalyst with excellent photocatalytic activity and stability, have been successfully synthesized through a simple two-step hydrothermal method. The related properties of the as-prepared photocatalyst were analyzed and a possible reaction mechanism was proposed. Incorporating the rareearth element $\mathrm{Er}$ into the $\mathrm{BiOBr}$ crystal lattice can greatly expand the optical window, maybe owing to the reduced energy band gap by the impurity energy level introduced below the conduction band and indirect utilization of long-wavelength visible light caused by the up-conversion performance. The wrapping with surface graphene nanosheets can efficiently promote charge separation and transmission over the hybrid photocatalyst. The synergistic effect between efficient solar energy harvesting and charge separation gives rise to a remarkable improvement of photocatalytic activity for RhB degradation under simulated sunlight irradiation. In addition, the as-prepared photocatalyst possesses excellent photocatalytic stability, mainly due to the robust coordination interaction between the graphene and the (001) facets of the $\mathrm{BiOBr}$ subunits. The rational design of this highly active and stable photocatalyst provides a promising approach for future applications.

Received 26th February 2017

Accepted 13th April 2017

DOI: $10.1039 / c 7 r a 02402 a$

rsc.li/rsc-advances

\section{Introduction}

Nowadays, semiconductor photocatalysts, such as $\mathrm{TiO}_{2}$, CdS, $\mathrm{MoS}_{2}, \mathrm{Bi}_{2} \mathrm{MoO}_{6}$ and $\mathrm{WO}_{3}$, which possess the ability of coupling solar energy into chemical reactions, have been widely investigated for solving some environmental and energy problems..$^{1-13}$ Nevertheless, the traditional photocatalysts have disadvantages, such as weak visible-light response and inefficient quantum efficiency, forcing us to develop new types of semiconductor photocatalytic materials. As a new type of photocatalyst, BiOX $(\mathrm{X}=\mathrm{F}, \mathrm{Cl}, \mathrm{Br}, \mathrm{I})$ are gaining increasing attention due to their unique layered structure, good optical properties and high photocatalytic activities. ${ }^{14,15}$ In particular, the band gap of BiOBr is the most reasonable. ${ }^{16,17}$ However, the pure BiOBr photocatalyst still faces some challenges which seriously limit its photocatalytic activity; thus, the further optimization of the conventional $\mathrm{BiOBr}$ photocatalyst is quite desirable and urgent. ${ }^{18-20}$ To our knowledge, the photocatalysis reaction process mainly contains three parts. ${ }^{21-23}$ The first part is solar energy harvesting, in which the semiconductor photocatalysts absorb solar energy and generate electrons and holes. The

School of Light Industry and Chemical Engineering, Dalian Polytechnic University, \#1 Qinggongyuan, Dalian 116034, P. R. China. E-mail: dongxl@dlpu.edu.cn second part is charge transfer, where photogenerated carriers migrate to the photocatalyst surface along with the charge recombination process. Finally, the surviving photocarriers over the photocatalyst surface can attack the targets. Thereby, realizing the efficient solar energy harvesting and charge separation can be considered as the effective pathway for improving the catalysis activity of photocatalyst. Illuminated by the aforesaid insight, introducing some foreign ions into BiOBr crystal or incorporating some cocatalysts into $\mathrm{BiOBr}$ photocatalysis system, which can efficiently boost the photoelectric conversion efficiency, holds great promise for realizing the overall improvement for the photocatalysis activity of the $\mathrm{BiOBr}$ catalyst. ${ }^{24-26}$

The introduction of foreign ions, namely ion doping, has been proven to be an effective way to improve the photocatalytic activity of BiOBr. ${ }^{27-30}$ To date, various experiments have been reported, some metal ions (e.g., $\mathrm{Zn}$ and Fe) or nonmetal (e.g., B and $\mathrm{C}$ ) doped $\mathrm{BiOBr}$ photocatalysts were successfully prepared $^{31-35}$ and exhibited good photocatalytic performance, which mainly attributed to several aspects, e.g. impurity energy level introduced into the energy band, internal electric field tuning and capture traps generated for electrons and holes. ${ }^{36,37}$ Besides that, the inherent nature of dopant also affects the catalytic activity of photocatalyst. ${ }^{38,39}$ Compared with 
conventional materials, the up-conversion materials possess the characteristic of converting low-energy photons into highenergy photons..$^{\mathbf{4 0 , 4 1}}$ Inspired this insight, incorporating appropriate amount of up-conversion materials which can indirectly utilize long-wavelength visible-light, holds great promise to extend the spectral response range of $\mathrm{BiOBr}$ and hence achieving the effective enhancement for its photocatalytic activity. Generally accepted, rare-earth elements mostly have up-conversion property, where the lanthanide Er possesses rich transition bands in the visible region, for example, at the wavelengths of $488 \mathrm{~nm}, 520 \mathrm{~nm}$ and $650 \mathrm{~nm} .{ }^{42}$ Therefore, we selected $\mathrm{Er}$ ions to coupled into the $\mathrm{BiOBr}$ crystal lattice, forming the synthetic of $\mathrm{Er}$ doped $\mathrm{BiOBr}$, to realize efficient utilization of solar energy.

Graphene, as a novel two-dimensional carbon material, possesses excellent electron mobility, superior optical transparency and high specific surface area. ${ }^{43-45}$ As the precursor of graphene, graphene oxide (GO) possesses abundant surface oxygen functional groups which enable it compound tightly with other materials and reduced to graphene simultaneously. ${ }^{46}$ Recently, using GO as the precursor, many BiOBr-graphene composites have been successfully synthesized, in which graphene was performed as an excellent co-catalyst. For example, Lee's group presents the composites via a simple solvothermal route. ${ }^{47}$ Zhang et al. successfully prepared the hybrids through a single-step microwave irradiation strategy. ${ }^{48}$ The composites showed good photocatalytic activity, owing to the efficient interface charge separation and transmission caused by the surface presence of graphene over BiOBr catalyst. ${ }^{49}$ In addition, the high flexibility of graphene and robust coordination interaction between graphene and $\mathrm{BiOBr}$ may give rise to high photocatalytic activity and stability of the photocatalyst.

For the first time, Er-doped BiOBr hollow microspheres wrapped with graphene nanosheets were successfully synthesized through a simple two-step hydrothermal method. Utilizing XRD, FT-IR, SEM, TGA, XPS, TEM, BET, UV-diffuse and PL techniques, a series of characterization of the samples were performed. In order to investigate the photocatalytic activity and stability of samples, photocatalytic degradation experiments under simulated visible-light were completed, using rhodamine $\mathrm{B}(\mathrm{RhB})$ solution as the target pollutants. Furthermore, the radicals trapping experiment was carried out and a possible mechanism for the photocatalytic reaction was put forward.

\section{Experimental}

\subsection{Materials}

2.1.1 Preparation of $\mathrm{BiOBr}$ hollow microspheres. In a typical process, $\mathrm{Bi}\left(\mathrm{NO}_{3}\right)_{3} \cdot 5 \mathrm{H}_{2} \mathrm{O}(240 \mathrm{mg})$ and $\mathrm{NaBr}(520 \mathrm{mg})$ were sequentially dissolved in $20 \mathrm{ml}$ boiled ethylene glycol solution (EG). After stirring for $30 \mathrm{~min}$, the above solution was added drop-wise to $60 \mathrm{ml}$ isopropanol (IPA) solution. Then, the mixture solution was sealed in $100 \mathrm{ml}$ Teflon-lined autoclave, heating at $160{ }^{\circ} \mathrm{C}$ for $12 \mathrm{~h}$. The product was collected, washed, and dried under room temperature for further characterization.
2.1.2 Preparation of Er-doped BiOBr hollow microspheres. Typically, the up-conversion element Er-doped BiOBr hollow microspheres were synthesized via a simple modification by adding appropriate amounts of $\operatorname{Er}\left(\mathrm{NO}_{3}\right)_{3} \cdot 5 \mathrm{H}_{2} \mathrm{O}$ into the IPA solution in advance. The mole ratios of $\mathrm{Er} / \mathrm{Bi}$ in as-prepared samples were $0.005,0.01,0.015$ and 0.02 , for convenience, which were marked as BE-0.5\%, BE-1\%, BE-1.5\% and $\mathrm{BE}-2 \%$ respectively.

2.1.3 Synthesis of graphite oxide. GO was prepared from pre-oxidation graphite powder (325 mesh) via a modified Hummers' method as previous published.

2.1.4 Fabrication of BE-1.5\% wrapped with graphene nanosheets. The hybrid photocatalysts of $\mathrm{BE}-1.5 \%$ wrapped with graphene nanosheets were prepared by a simple hydrothermal method. The mixture of $\mathrm{BE}-1.5 \%$ and appropriate amounts of GO aqueous solution was vigorous stirred for $4 \mathrm{~h}$, then transferred to $100 \mathrm{ml}$ Teflon-lined autoclave, to fill up to $80 \%$ of the total volume and, heated at $100{ }^{\circ} \mathrm{C}$ for $12 \mathrm{~h}$. After filtrating, washing and drying, the final grey products were collected for further characterization. The mass ratio of GO and BE-1.5\% were $1 \%, 3 \%, 5 \%$ respectively. According to the mass ratio, the as-prepared photocatalysts were marked as BE@GR-1, BE@GR-3, BE@GR-5, respectively.

\subsection{Characterization of the catalysts}

The crystalline structure of the samples was characterized via a Shimadzu XRD-6100 diffractometer. Fourier transform infrared spectroscopy (FT-IR, Nicolet 380) was used to determine the surface functional groups. The sample morphology was obtained using a field emission scanning electron microscope (JSM-7800F, JEOL) equipped with an energy dispersive analysis system of X-ray (EDX) and elemental mapping system. X-ray photoelectron spectroscopy (XPS) measurement by a Thermo VG ESCALAB-250 system. TEM images were performed by a JEOL JEM-2100F transmission electron microscope. UV-Vis diffuse reflectance spectra of the samples were measured by UV-Vis spectrophotometer (CARY100\&300, VARIAN). The PL spectra of the photocatalysts were obtained using Hitachi F7000 fluorescence spectrophotometer. The TGA analysis was performed by the PerkinElmer diamond TG/DTA analyzer (Japan) at a heating rate of $10{ }^{\circ} \mathrm{C} \mathrm{min}^{-1}$. The $\mathrm{N}_{2}$-sorption measurement was performed by using Micromeritics Tristar 3000 at $77 \mathrm{~K}$, the specific surface area and the pore size distribution were estimated using the Brunauer-Emmett-Teller (BET) and BarrettJoyner-Halenda (BJH) methods, respectively.

\subsection{Photocatalytic test}

The photocatalytic activity is proportional to the photocatalytic degradation rate, where we adopt $\mathrm{RhB}$ solution as the target pollutant. Typically, under simulated sunlight, the photocatalyst (20 mg) was dissolved into RhB solution $\left(10 \mathrm{mg} \mathrm{l}^{-1}\right)$. First, the mixture was stirred for $40 \mathrm{~min}$ to achieve adsorption/desorption equilibrium without light irradiation. Then, under illumination, the mixture was sampled every five minutes and centrifuged to remove the photocatalyst. Finally, the samples were quantitatively analyzed by using UV-Vis spectrophotometer. Afterwards, 
the degradation efficiency (\%) was measured, using the following formula:

$$
\text { Degradation efficiency }(\%)=\left(C_{0}-C\right) / C_{0} \times 100 \%
$$

where $C_{0}$ is the initial concentration of $\mathrm{RhB}$ and $C$ is the timedependent concentration of dye upon irradiation. Furthermore, the degradation efficiency (\%) in photocatalytic cycle experiments was obtained using the same process.

\section{Results and discussion}

\subsection{XRD analysis}

$\mathrm{X}$-ray diffraction (XRD) is an effective way to study the crystal structure. The XRD patterns of BiOBr, BE-1.5\% and BE@GR-3 were demonstrated (Fig. 1). Seven distinct diffraction peaks of the samples at $10.9^{\circ}, 25.3^{\circ}, 32.3^{\circ}, 39.4^{\circ}, 46.3^{\circ}, 57.3^{\circ}$ and $67.6^{\circ}$ indexed to (001), (011), (110), (112), (020), (212) and (220) crystal planes (JCPDS 73-2061) of tetragonal phase BiOBr, indicating the successful synthesis of BiOBr-based hybrids. The peaks of Er ion and graphene are not found in the BE-1.5\% and BE@GR-3, attributing to the low-amount of the species. For the samples modified with Er ions, their phases keep the same with pure BiOBr. However, the peaks indexed at (001) can be found with slight shift. In addition, the peaks intensity of $\mathrm{BE}-1.5 \%$ and BE@GR-3 are lower than that of pristine BiOBr. The poor intensity and slight shift of diffraction peaks maybe ascribed to the introduction of element $\mathrm{Er}$ into BiOBr crystal lattice, which causing the crystal lattice defects of BiOBr. Focusing on (001) crystal planes, BE@GR-3 displays a lower peak intensity compared with BE-1.5\%, suggesting the formation of coordination interaction between graphene and the (001) facets of BiOBr subunits. ${ }^{50}$ In summary, the hybrid photocatalyst (Erdoped BiOBr wrapped with graphene) was successfully synthesized.

\subsection{FT-IR spectra analysis}

The information of functional groups or chemical bonds over the catalysts was analyzed by FT-IR spectra. Fig. 2 shows the FTIR spectra of GO, BiOBr, BE-1.5\% and BE@GR-3, marked as a, b, $\mathrm{c}$, $\mathrm{d}$ respectively. The absorption band at $510 \mathrm{~cm}^{-1}$, as evidenced in the spectrum of BiOBr, BE-1.5\% and BE@GR-3, is classified as the $\mathrm{Bi}-\mathrm{O}$ functional group. Besides that, the absorption peaks



Fig. 1 XRD patterns of $\mathrm{BiOBr}, \mathrm{BE}-1.5 \%$ and $\mathrm{BE}(\mathrm{GR}-3$.



Fig. 2 FT-IR spectra of (a) GO, (b) BiOBr, (c) BE-1.5\% and (d) BE(aGR-3.

of BE@GR-3 consist of O-H stretching vibration $\left(3540 \mathrm{~cm}^{-1}\right)$, $\mathrm{C}=\mathrm{C}$ stretching vibration and $\mathrm{O}-\mathrm{H}$ bending vibration (1600 $\left.\mathrm{cm}^{-1}\right)$, and carboxylates $\mathrm{C}-\mathrm{O}$ stretching vibration $\left(1120 \mathrm{~cm}^{-1}\right)$. Specially, the $\mathrm{C}-\mathrm{O}$ stretching peak at $1120 \mathrm{~cm}^{-1}$ is found in BE@GR-3 and bare GO, which is absent in the BiOBr and BE$1.5 \%$, indicating the successful synthesis of the BiOBr wrapped with graphene. The $\mathrm{C}=\mathrm{O}$ stretching vibration $\left(1727 \mathrm{~cm}^{-1}\right)$ and other characteristic peaks of GO are not found in the BiOBrbased catalysts, revealing that the reduction of GO. The peak at $1380 \mathrm{~cm}^{-1}$ for pristine $\mathrm{BiOBr}$ is classified as carboxyl $\mathrm{O}-\mathrm{H}$ stretching vibration $\left(1400 \mathrm{~cm}^{-1}\right)$, which is not found in BE$1.5 \%$, owing to the introduction of element Er into BiOBr crystal lattice.

\subsection{SEM analysis}

The SEM images of BiOBr, BE-1.5\% and BE@GR-3 were displayed (Fig. 3). We can see that the pristine BiOBr composed of quasi microspheres with a diameter of 1-2 $\mu \mathrm{m}$ (Fig. 3a). The enlarged view of BiOBr shows the microspheres with hollow structure assembled by irregular nanosheets (Fig. 3b). Compared with the pristine $\mathrm{BiOBr}$, the morphology of $\mathrm{BE}-1.5 \%$ basically unchanged (Fig. 3c), and the hollow structure remained (Fig. 3d). The results demonstrate there is no obvious effect on the morphology structure of the catalyst by incorporating element Er into the BiOBr crystal lattice. Fig. 3e shows the graphene nanosheets with many wrinkles surface coated on BE$1.5 \%$ hollow microsphere. High magnification SEM image of

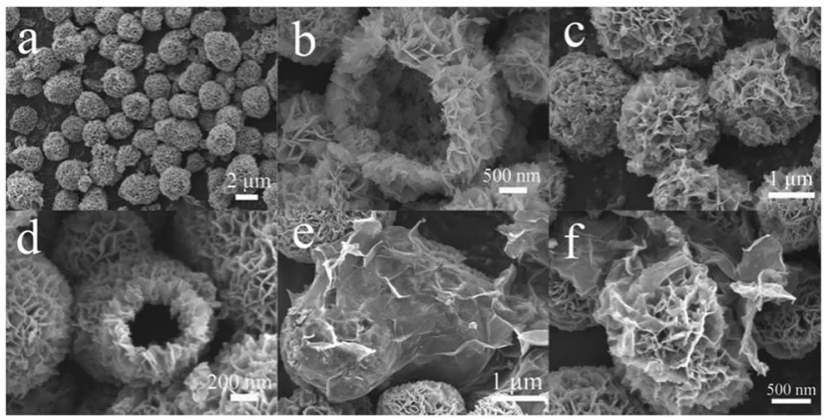

Fig. 3 SEM images with different magnification of (a and b) $\mathrm{BiOBr}$, (c and d) BE-1.5\% and (e and f) BE(aGR-3. 
BE@GR-3 further determines the hollow microspheres BE-1.5\% wrapped with graphene nanosheets (Fig. 3f). The SEM images clearly show the successful formation of Er-doped BiOBr hollow microspheres wrapped with graphene nanosheets.

\subsection{EDS analysis}

The type and distribution of typically elements from the samples were received from EDS mapping images. Fig. 4a-c represent the EDS mapping images of $\mathrm{BiOBr}, \mathrm{BE}-1.5 \%$ and BE@GR-3 respectively. From Fig. 4a, the elements Bi, O and Br are distributed homogeneously within individual microspheres, suggesting the successful synthesis of BiOBr hollow microspheres. The morphology of $\mathrm{BE}-1.5 \%$ remains unchanged comparison with the pristine $\mathrm{BiOBr}$, however, the chemical component is indeed altering after incorporating the element Er into BiOBr matrix (Fig. 4b). The element Er is distributed homogeneously with the element $\mathrm{Bi}$ and $\mathrm{Br}$, indicating the successful synthesis of Er-doped BiOBr. Fig. 4c shows the focus of graphene nanosheets which are deposited over the BE-1.5\% microspheres, identified as element $\mathrm{C}$, which is in agreement with the conclusion of Er-doped BiOBr microspheres wrapped with graphene nanosheets.

\subsection{Thermogravimetric analysis (TGA)}

TGA measurement was used to evaluate the weight percentage of graphene in BE@GR-3. As can be seen in Fig. 5, the first weight loss in the temperature range from 20 to $150{ }^{\circ} \mathrm{C}$ is mainly assigned to the de-intercalation or removal of moisture content, mainly water molecules. The second major weight loss centered at $200-530{ }^{\circ} \mathrm{C}$ was mainly ascribed to the removal of oxygen containing functional groups and the decomposition of carbon skeleton in composite, which is corresponding to the loss of graphene $(\sim 3.0 \%)$, consistent with the experimental value. Finally, when the BE@GR-3 composite was heated to $650{ }^{\circ} \mathrm{C}$, the residual mass was calculated as $81 \%$, which reveals the good thermal stability of photocatalyst. ${ }^{51,52}$ The main reason was the strong interaction between the GO and the BiOBr matrix that made the composite highly stable.



Fig. 4 SEM images and EDS mappings of (a) $\mathrm{BiOBr}$ with $\mathrm{Bi}, \mathrm{Br}, \mathrm{O}$ elemental maps, (b) BE-1.5\% with $\mathrm{Bi}, \mathrm{Br}$, Er elemental maps and (c) BE@GR-3 with C, Bi, Er elemental maps.



Fig. 5 TGA spectrum of BEaGR-3 composite.

\subsection{XPS analysis}

The surface element composition of BE@GR-3 was further studied by X-ray photoelectron spectroscopy (XPS), and the corresponding chemical states were received. The XPS survey spectra of BE@GR-3 reveals that the sample is composed of elements of $\mathrm{Bi}, \mathrm{Br}, \mathrm{Er}, \mathrm{O}$ and $\mathrm{C}$ (Fig. 6a), which is according with the chemical composition of photocatalyst. The corresponding chemical states of elements were respectively investigated in Fig. 6b-f. Two strong peaks at 159.1 and $164.5 \mathrm{eV}$ in the highresolution $\mathrm{Bi}$ 4f spectra are assigned to $\mathrm{Bi} 4 \mathrm{f}_{7 / 2}$ and $\mathrm{Bi} 4 \mathrm{f}_{5 / 2}$, which are characteristic of the $\mathrm{Bi}^{3+}$ in $\mathrm{BE} @ G \mathrm{G}-3$ sample (Fig. 6b). Meanwhile, the peak with binding energy of $68.4 \mathrm{eV}$ (Fig. 6c), belonged to $\mathrm{Br} 3 \mathrm{~d}$, is the characteristic of $\mathrm{Br}^{-}$in the photocatalyst. The peak in Er 4d spectra (Fig. 6d) at $168.6 \mathrm{eV}$ is consistent with erbium in $\mathrm{Er}_{2} \mathrm{O}_{3},{ }^{53}$ revealing the existence of $\mathrm{Er}^{3+}$ in the sample. As shown in Fig. 6e, the spectrum for O 1s can be fitted to two peaks at 529.9 and $531.9 \mathrm{eV}$, which are ascribed to the lattice oxygen and surface hydroxyl groups of catalyst, respectively. The low intensity of surface hydroxyl groups is compatible with the results of FT-IR analysis. The C 1s spectra centered at $284.6 \mathrm{eV}$ as reference can be deconvoluted to two peaks at 284.5 and $286.3 \mathrm{eV}$, which are assigned to the typical carbon-carbon $(\mathrm{C}-\mathrm{C})$ bonds and $\mathrm{C}-\mathrm{O}$ bonds, respectively. ${ }^{54}$ Besides that, the peak of $\mathrm{C}=\mathrm{O}$ bonds around $288.4 \mathrm{eV}$ is also not found, further verifying the FT-IR analysis.

\subsection{TEM images analysis}

The TEM images further determine above morphology and microstructure analysis. Fig. 7a shows the TEM image of $\mathrm{BiOBr}$
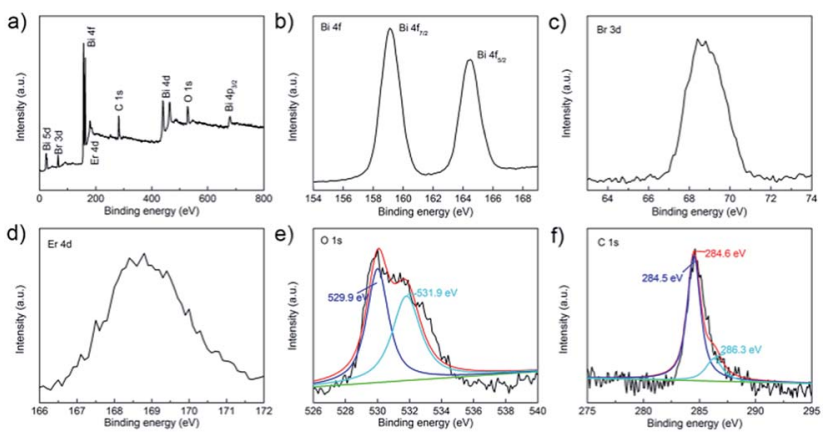

Fig. 6 XPS spectra of the as-prepared BE@GR-3: (a) survey, (b) Bi 4f, (c) $\mathrm{Br} 3 \mathrm{~d}$, (d) $\mathrm{Er} \mathrm{4d,} \mathrm{(e)} \mathrm{O} \mathrm{1s,} \mathrm{and} \mathrm{(f)} \mathrm{C} \mathrm{1s.}$ 


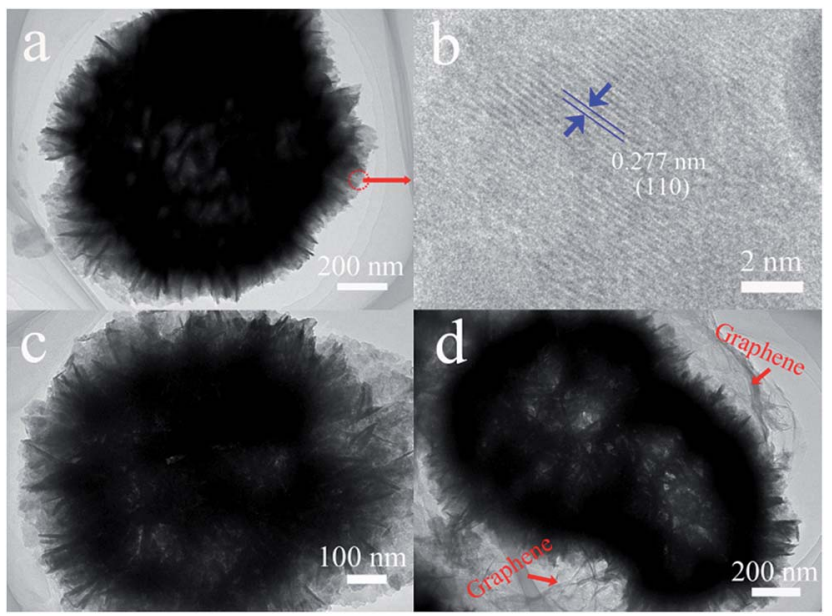

Fig. 7 TEM images of (a) BiOBr, (c) BE-1.5\%, (d) BEaGR-3 and HRTEM image of (b) $\mathrm{BiOBr}$.

with black round edge and bright center, further evidencing the hollow microsphere structure of BiOBr. Besides that, the black round edge is inserted with irregular nanosheets, which is well consistent with the SEM images of BiOBr. The HRTEM image of BiOBr (Fig. 7b) shows crystals with interplanar spacing of $0.277 \mathrm{~nm}$, which corresponding to the (110) spacing of BiOBr. The above results can infer the conclusion of (001) dominant exposed-facet of BiOBr. ${ }^{55}$ The TEM image of $\mathrm{BE}-1.5 \%$ is similar with the pristine $\mathrm{BiOBr}$, which is according with the SEM analysis (Fig. 7c). As shown in the image of BE@GR-3 (Fig. 7d), ultrathin mist-like graphene with distinct wrinkle were anchored on the hollow microsphere can be clearly observed, confirming the synthesis of BiOBr hollow microspheres wrapped with graphene nanosheets.

\subsection{BET analysis}

Fig. 8 shows the nitrogen sorption isotherms of BiOBr, BE-1.5\% and BE@GR-3, respectively. All the nitrogen sorption isotherms exhibit type IV classification with typical hysteresis loops in the relative pressure $\left(P / P_{0}\right)$ range of $0.45-0.95$, which belong to mesopore characteristics. The pore sizes of the samples were

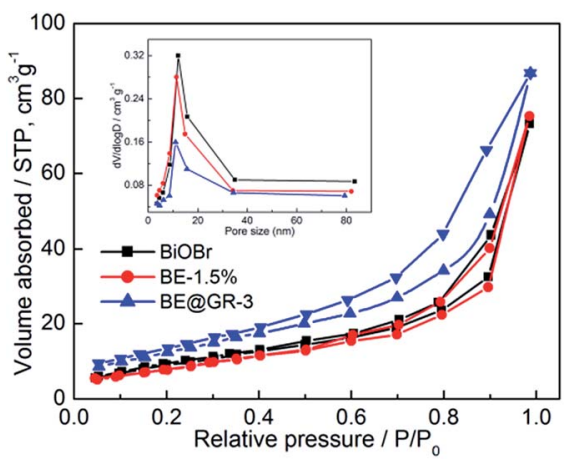

Fig. 8 Nitrogen adsorption-desorption isotherms of $\mathrm{BiOBr}, \mathrm{BE}-1.5 \%$ and $\mathrm{BE} @ \mathrm{GR}-3$; inset: the corresponding pore-size distribution. calculated to be $11 \mathrm{~nm}$, using the Barrette-Joynere-Halenda (BJH) method (Fig. 8 inset). The Brunauer-Emmett-Teller (BET) surface areas are about 29,27 and $43 \mathrm{~m}^{2} \mathrm{~g}^{-1}$ for the pristine BiOBr, BE-1.5\% and BE@GR-3, respectively. The specific surface area of $\mathrm{BE}-1.5 \%$ is generally equaled with the pristine $\mathrm{BiOBr}$, confirming the remained of morphology and structure. The increase in the surface area of the BE@GR-3 composites mainly results from the addition of the graphene nanosheets with a low density and high surface area, which may offer a large interface to facilitate the adsorption and catalytic performance.

\subsection{Formation mechanism}

Based on the above experimental results, the BiOBr-based catalysts with hollow sphere structure are indeed synthesized and a possible growth pattern and formation mechanism is shown in Scheme 1. First, the alkoxide complexes formed by $\mathrm{Bi}^{3+}$ cations and EG lead to a slow nucleation of $\mathrm{BiOBr}$, which tend to assemble into spherical agglomerates driven by reducing the surface energy of the nanoparticles (step 1 in Scheme 1). ${ }^{56}$ To our knowledge, the BiOBr crystalline with tetragonal matlockite structure are constructed by $\left[\mathrm{Bi}_{2} \mathrm{O}_{2}\right]$ slabs interleaving double slabs of bromine atoms, which is partly similar to $\mathrm{Bi}_{2} \mathrm{WO}_{6}$ and $\mathrm{Bi}_{2} \mathrm{MoO}_{6} \cdot{ }^{57,58}$ Then the solution environment is weakly acidic, the smaller crystallites in the spherical agglomerates are gradually dissolved and $\mathrm{Bi}_{2} \mathrm{O}_{2}{ }^{2+}$ and $\mathrm{Br}^{-}$ ions are generated under solvothermal conditions because of the concentration difference between the solution and the surface of the microspheres, which is similar to the previous research. ${ }^{59}$ And the ions of $\mathrm{Bi}_{2} \mathrm{O}_{2}{ }^{2+}$ and $\mathrm{Br}^{-}$in the solution preferential recrystallization on the small protuberances of spherical surface, which provide many high-energy sites for nanocrystalline growth. ${ }^{60}$ Then, these self-agglomerate spheres would further dissolve and recrystallize to generate $\mathrm{BiOBr}$ nanosheets assembled into hollow microspheres for high intrinsic anisotropic properties. Thus, hierarchical $\mathrm{BiOBr}$ hollow microspheres are synthesized because of mass diffusion and Ostwald ripening (step 2 in Scheme 1). And, few Er ions doping not obviously affect the growth process, as performed by the experiment result. Finally, the Er-doped BiOBr hollow microspheres wrapped with graphene nanosheets were synthesized via a simple hydrothermal method (step 3 in

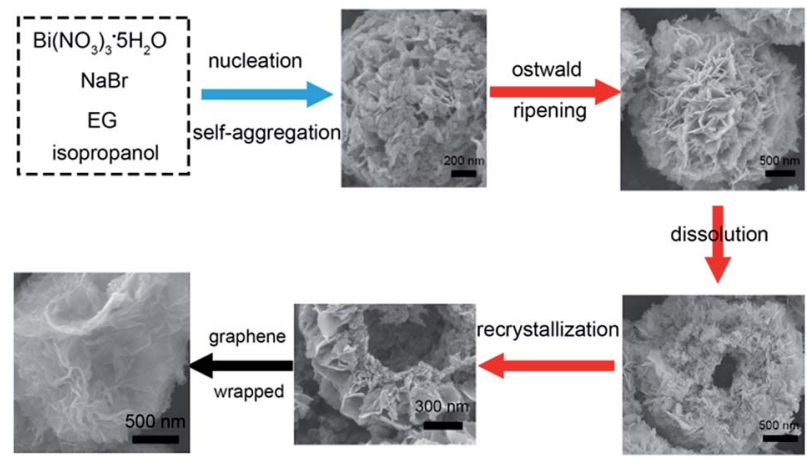

Scheme 1 The formation mechanism of Er-doped BiOBr hollow microspheres wrapped with graphene nanosheets. 
Scheme 1), using CTAB as the surfactant, which is inspired by Zhao's study. ${ }^{61}$

\subsection{UV-Vis spectroscopy analysis}

The optical absorption property of samples was tested by UV-Vis diffuse reflectance spectra. The spectra of the samples with different concentrations of $\mathrm{Er}$ element were put forward (Fig. 9a). After incorporating element Er into BiOBr crystal lattice, obvious shift of the photoabsorption edges can be found. On the other hand, the intensity and range of the photoabsorption were also significantly improved. Furthermore, the lower absorbance of BE-2\% compared with BE-1\% and BE$1.5 \%$, which is contrary with the doping amount maybe owing to the resulting poor crystallization. In summary, $1.5 \%$ mole ratio doping amount endowed the as-prepared sample with best optical absorption property. Interestingly, two characteristic absorption peaks at the wavelength of $520 \mathrm{~nm}$ and $650 \mathrm{~nm}$ were found (Fig. 9a inset), which were not found in the pristine $\mathrm{BiOBr}$ and other BiOBr-based hybrids. This optical phenomenon can be ascribed to the up-conversion effect of rare earth element Er. Besides that, the energy band gap can be evaluated through using the eqn (2).

$$
\alpha h \nu=A\left(h \nu-E_{\mathrm{g}}\right)^{1 / 2}
$$

The energy band gap ( $E_{\mathrm{g}}$ values) of $\mathrm{BiOBr}$ and $\mathrm{BE}-1.5 \%$ were estimated to be about $2.7 \mathrm{eV}$ and $2.45 \mathrm{eV}$ respectively (Fig. 9b), suggesting the impurity energy level introduced below the conduction band. The reduced energy band gap and indirect utilization of long-wavelength visible-light were simultaneously achieved by the novel introduction of up-conversion element $\mathrm{Er}$ into the BiOBr photocatalyst.
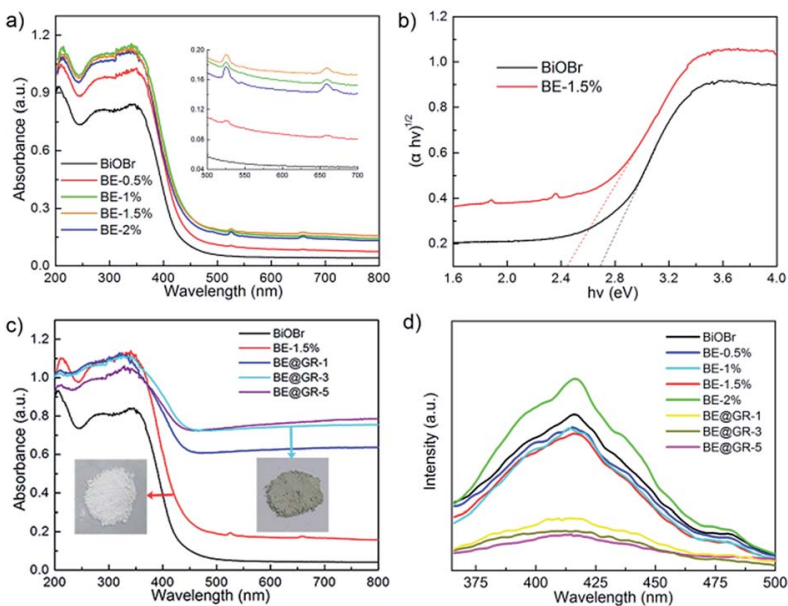

Fig. 9 (a) UV-Vis diffuses reflectance spectra of the Er-doped $\mathrm{BiOBr}$ samples with different $\mathrm{Er}$ values and (inset) the corresponding part ranging from $500 \mathrm{~nm}$ to $700 \mathrm{~nm}$ and (c) BE-1.5\% wrapped with different amounts of graphene nanosheets and (inset) digital photographs of catalyst grains of BE-1.5\% and BE@GR-3, (b) curves of $(\alpha h \nu)^{1 / 2}$ versus photon energy $(h \nu)$ of the as-prepared samples and (d) the PL spectra of as-prepared samples.
The spectral responsive property of the BE-1.5\% wrapped with graphene nanosheets was investigated (Fig. 9c). No evident shift of the photoabsorption edges of the samples are found, revealing the graphene is surface wrapping the photocatalyst, which is consistent with the FT-IR analysis. The absorbance at the wavelength ranging from $200 \mathrm{~nm}$ to $450 \mathrm{~nm}$ for $\mathrm{BE}-1.5 \%$, BE@GR-1, BE@GR-3 and BE@GR-5 is similar. The high absorbance in the tail region can be ascribed to the dark background owing to the graphene nanosheets wrapping. The optical property has been reported in other hybrid photocatalysts coupling with graphene. ${ }^{\mathbf{6 2 6 3}}$ In summary, the incorporated graphene is not essentially impacting the solar energy utilization.

\subsection{PL spectrum analysis}

The separation efficiency of photogenerated electrons and holes was investigated by photoluminescence emission spectra (PL spectra) (Fig. 9d). Generally accepted, high fluorescence intensity represents the high recombination rate of photocarriers which gives rise to low photocatalytic activity. The fluorescence intensity of samples were $\mathrm{BE}-2 \%$, BiOBr, BE-0.5\%, BE- $1 \%$, BE1.5\%, BE@GR-1, BE@GR-3 and BE@GR-5 from top to bottom, and the last three were significant lower than that of the others. It is worth noted that minor Er elements doping slightly decreased the fluorescence intensity of catalysts, indicating the enhanced charge separation caused by the impurity energy level incorporating. However, excess impurity ions are incorporated into the photocatalyst which become new charge recombination centers, resulting in a weak charge separation and strong fluorescence effect. After coupling with graphene nanosheets, the fluorescence intensity of photocatalyst was obviously decreased, confirming the incorporated graphene into the photocatalysis system is an effective pathway to repress the recombination of photocarriers and thus resulting efficient charge transfer, which is very beneficial for the enhancement of photocatalytic activity.

\subsection{Photocatalytic activity analysis}

Fig. 10 shows the photocatalyst degradation performance over BiOBr-based samples, using RhB as the target pollution. It is found that the self-photodegradation of $\mathrm{RhB}$ is almost negligible and the Er-doped BiOBr samples have higher photocatalytic degradation rates (Fig. 10a), confirming the incorporated Er ions have a good effect on the photocatalytic activity. Furthermore, BE-1.5\% possesses the best photocatalytic activity, according with the above property analysis. The influence of coupling graphene on the photocatalytic degradation rate was explored (Fig. 10b). Interestingly, the dye absorption performances are significantly increased with graphene wrapping, and when BE$1.5 \%$ wrapped with $3 \mathrm{wt} \%$ graphene nanosheets exhibits the most excellent photocatalytic activity. To directly observe the photocatalytic activity, the photocatalytic degradation kinetics equation was using (Fig. 10c),

$$
-\ln \left(C_{0} / C_{t}\right)=k t
$$

where $C_{0}$ and $C_{t}$ are the concentrations of RhB at times 0 and $t$, respectively, and $k$ is the apparent first-order rate constant 



Fig. 10 Variation of $\mathrm{RhB}$ concentration against irradiation time using (a) Er-doped $\mathrm{BiOBr}$ samples with different REr values, (b) BE-1.5\% wrapped with different concentrations of $\mathrm{GO},(\mathrm{c})-\ln \left(C_{t} / C_{0}\right)$ versus irradiation time and (d) the rate constant $k$ of the degradation of RhB in the presence of different catalysts.

$\left(\min ^{-1}\right)$. The corresponding $k$ values of samples incorporated with different amounts of Er element and graphene were calculated to be $0.013,0.01944,0.02599,0.03007,0.01748$, $0.03565,0.04658$ and $0.02697 \mathrm{~min}^{-1}$ respectively (Fig. 10d). BE$1.5 \%$ possesses the maximum $k$ value in the Er-doped $\mathrm{BiOBr}$ samples, which is almost 2.3 times higher than that of the pristine BiOBr. In addition, the BE@GR-3 exhibits the best photocatalytic decomposition efficiency.

\subsection{Radical trapping experiments}

To investigate the surface reaction mechanism of BE@GR-3, radical trapping experiments were performed. In the typical degradation experiment, three radical scavengers, isopropanol (IPA), 1,4-benzoquinone (BQ) and methanol as the scavengers of $\cdot \mathrm{OH},{ }^{\circ} \mathrm{O}_{2}{ }^{-}$radicals and $\mathrm{h}^{+}$, were added into the degradation process system respectively, and the results were shown in Fig. 11a.

As shown, the degradation efficiency of BE@GR-3 with the addition of IPA is nearly coincident with that of the control group, implying ${ }^{\circ} \mathrm{OH}$ played little influence upon the reaction. Moreover, higher curves with the presence of methanol and BQ represent the low photocatalytic activity. It can be inferred that both $\mathrm{h}^{+}$and ${ }^{\circ} \mathrm{O}_{2}{ }^{-}$play key roles in the photocatalytic degradation reaction.

\subsection{Photocatalytic stability tests}

The excellent photocatalyst should possess high photocatalytic stability for future application. To study the photocatalytic stability of the BE@GR-3, photocatalytic cycle reaction was carried out, and the results were shown in Fig. 11b. After five cycles, the degradation rate of the catalyst is slightly decreased, maybe ascribed to the loss of photocatalyst in the washing stage. The XRD patterns of BE@GR-3 before and after the five
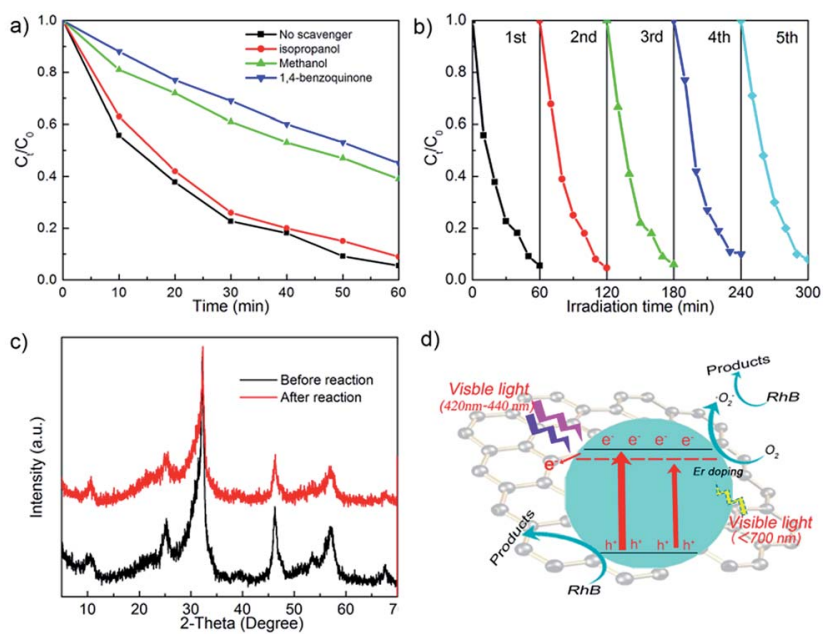

d)

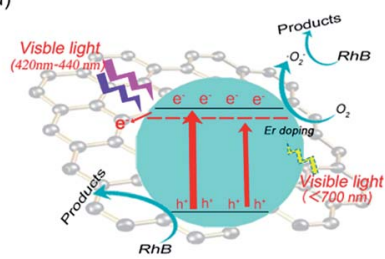

Fig. 11 (a) Radical trapping experiments over BEaGR-3 using different scavengers, (b) recycling tests over BE@GR-3 for the degradation of RhB under visible light irradiation, (c) XRD diffraction patterns of the BE(aGR-3 before and after five cycles and (d) proposed mechanism for the photocatalytic degradation of RhB over BEAGR-3 under visiblelight irradiation.

cycles were demonstrated (Fig. 11c), which are basically unchanged, further confirming the excellent stability.

\subsection{Proposed mechanism}

Herein, we propose a possible mechanism for the photocatalytic degradation process (Fig. 11d). Based on the experiment results, BiOBr hollow microspheres wrapped with graphene nanosheets wrapping possess large specific surface area, resulting with strong dye adsorption performance and abundant surface reaction sites. Under simulated sunlight irradiation, the $\mathrm{BiOBr}$ microspheres with hollow structure assembled by nanosheets make multiple scattering for incident light, realizing the better solar energy harvesting. ${ }^{3,4}$ Following, BiOBr photocatalyst can convert the absorbed photons into energetic electrons and holes, respectively. The introduction of up-conversion element Er into the BiOBr crystal lattice gives rises to impurity energy level introduced below the conduction band. The impurity energy level paves a shorter channel for the energy conversion, leading to the broadened photoresponse range. In addition, the dramatic photoresponse at the wavelength around $520 \mathrm{~nm}$ and $650 \mathrm{~nm}$ of photocatalyst was found, due to the up-conversion property of rare-earth element Er. That is, the introduction of element Er endows BiOBr matrix the ability to absorb the nearly all visible light, which greatly broaden the optical window, resulting the efficient solar energy harvesting. As a result, the efficient solar energy harvesting leads to the more photoinduced electrons (or holes) generated. Then, the electrons can be captured and transferred rapidly by the graphene nanosheets, thus greatly suppressing the surface charge recombination. The active electrons on the surface of catalysts can react with adsorbed $\mathrm{O}_{2}$ and generate ${ }^{\circ} \mathrm{O}_{2}{ }^{-}$, in which ${ }^{\circ} \mathrm{O}_{2}{ }^{-}$can decompose the RhB. On the other hand, the photoinduced holes can directly oxidize the RhB. The synergistic effect between efficient 
solar energy harvesting and charge separation is contributed to the high photocatalyst activity. Moreover, the robust coordination interaction between graphene and the (001) facets of BiOBr subunits, enables the remarkable photocatalytic stability for repeated utilization of photocatalyst.

\section{Conclusion}

In summary, we successfully synthesized the novel photocatalyst of Er-doped BiOBr hollow microspheres wrapped with graphene nanosheets, which possesses excellent photocatalytic activity and stability. The Er-doped BiOBr hollow microspheres possess efficient solar energy harvesting, attributing to two aspects, one is the reduced energy band gap by introducing impurity energy level into the energy band, and the other is indirectly utilizing long-wavelength visible-light caused by upconversion effect. The sample wrapped with graphene nanosheets displays better photocatalytic activity, owing to the efficient interface charge separation and transmission. What's more, the high photocatalytic stability of as-prepared sample maybe ascribed to the robust coordination interaction between graphene and the (001) facets of $\mathrm{BiOBr}$ subunits. This work sheds light on the comprehensive promoting the photocatalytic activity by means of a rational design.

\section{Acknowledgements}

This research was supported by the National Natural Science Foundation of China (Grant no. 21476033 and 21577008).

\section{Notes and references}

1 Z. Xiong, H. B. Wang, N. Y. Xu, H. L. Li, B. Z. Fang, Y. C. Zhao, J. Y. Zhang and C. G. Zheng, Int. J. Hydrogen Energy, 2015, 40, 10049-10062.

2 S. Q. Fan, C. Kim, B. Z. Fang, K. X. Liao, G. J. Yang, C. J. Li, J. J. Kim and J. Ko, J. Phys. Chem. C, 2011, 115, 7747-7754.

3 B. Z. Fang, A. Bonakdarpour, K. Reilly, Y. Xing, F. Taghipour and D. P. Wilkinson, ACS Appl. Mater. Interfaces, 2014, 6, 15488-15498.

4 B. Z. Fang, Y. Xing, A. Bonakdarpour, S. C. Zhang and D. P. Wilkinson, ACS Sustainable Chem. Eng., 2015, 3, 2381-2388.

5 Z. Z. Wu, B. Z. Fang, Z. P. Wang, C. L. Wang, Z. H. Liu, F. Y. Liu, W. Wang, A. Alfantazi, D. Z. Wang and D. P. Wilkinson, ACS Catal., 2013, 3, 2101-2107.

6 Z. Z. Wu, C. Y. Tang, P. Zhou, Z. H. Liu, Y. S. Xu, D. Z. Wang and B. Z. Fang, J. Mater. Chem. A, 2015, 3, 13050-13056.

7 K. Honda and A. Fujishima, Nature, 1972, 238, 37-38.

8 J. Schneider, M. Matsuoka, M. Takeuchi, J. Zhang, Y. Horiuchi, M. Anpo and D. W. Bahnemann, Chem. Rev., 2014, 114, 9919-9986.

9 E. M. El-Menyawy, I. T. Zedan and A. A. Azab, J. Alloys Compd., 2017, 695, 3429-3434.

10 R. X. Zhao, X. P. Li, J. X. Su and X. H. Gao, Appl. Surf. Sci., 2017, 392, 810-816.
11 Y. C. Hao, X. L. Dong, S. R. Zhai, X. Y. Wang, H. C. Ma and X. F. Zhang, Chem.-Eur. J., 2016, 22, 1-8.

12 Y. C. Hao, X. L. Dong, S. R. Zhai, X. Y. Wang, H. C. Ma and X. F. Zhang, RSC Adv., 2016, 6, 35709-35718.

13 Y. C. Hao, X. L. Dong, X. Y. Wang, H. C. Ma and X. F. Zhang, RSC Adv., 2016, 6, 102155-102158.

14 J. Li, Y. Yu and L. Z. Zhang, Nanoscale, 2014, 6, 8473-8488.

15 M. Shang, W. Z. Wang and L. Zhang, J. Hazard. Mater., 2009, 167, 803-809.

16 W. B. Li, Y. P. Zhang, Y. Y. Bu and Z. Y. Chen, J. Alloys Compd., 2016, 680, 677-684.

17 C. Q. Xu, H. H. Wu and F. L. Gu, J. Hazard. Mater., 2014, 275, 185-192.

18 F. F. Duo, Y. W. Wang, C. M. Fan, X. C. Zhang and Y. F. Wang, J. Alloys Compd., 2016, 685, 34-41.

19 L. Lin, M. H. Huang, L. P. Long and D. H. Chen, J. Alloys Compd., 2014, 615, 929-932.

20 Y. C. Feng, L. Li, J. W. Li, J. F. Wang and L. Liu, J. Hazard. Mater., 2011, 192, 538-544.

21 S. Bai, J. Jiang, Q. Zhang and Y. J. Xiong, Chem. Soc. Rev., 2015, 44, 2893-2939.

22 S. Q. Liu, Z. R. Tang, Y. G. Sun, J. C. Colmenares and Y. J. Xu, Chem. Soc. Rev., 2015, 44, 5053-5075.

23 D. Robert and S. Malato, Sci. Total Environ., 2002, 291, 85-97. 24 X. M. Zhang, G. B. Ji, Y. S. Liu, X. G. Zhou, Y. Zhu, D. N. Shi, P. Zhang, X. Z. Cao and B. Y. Wang, Phys. Chem. Chem. Phys., 2015, 17, 8078-8086.

25 Y. C. Hao, X. L. Dong, S. R. Zhai, X. Y. Wang, H. C. Ma and X. F. Zhang, Chem. Commun., 2016, 52, 6525-6528.

26 Y. C. Hao, X. L. Dong, S. R. Zhai, X. Y. Wang, H. C. Ma and X. F. Zhang, J. Mater. Chem. A, 2016, 4, 8298-8307.

27 X. Li, G. H. Jiang, Z. Wei, X. H. Wang, W. X. Chen and L. Shen, MRS Commun., 2013, 3, 219-224.

28 Z. S. Liu, B. T. Wu, Y. L. Zhao, J. N. Niu and Y. B. Zhu, Ceram. Int., 2014, 40, 5597-5603.

29 B. Pare, B. Sarwan and S. B. Jonnalagadda, Appl. Surf. Sci., 2011, 258, 247-253.

30 R. Wang, G. Jiang, X. Wang, R. Hu, X. Xi, S. Bao, Y. Zhou, T. Tong, S. Wang, T. Wang and W. Chen, Powder Technol., 2012, 228, 258-263.

31 G. H. Jiang, X. H. Wang, Z. Wei, X. Li, X. G. Xi, R. B. Hu, B. L. Tang, R. J. Wang, S. Wang, T. Wang and W. X. Chen, J. Mater. Chem. A, 2013, 1, 2406-2410.

32 D. Wu, S. T. Yue, W. Wang, T. C. An, G. Y. Li, H. Y. Yip, H. J. Zhao and P. K. Wong, Appl. Catal., B, 2016, 192, 35-45.

33 J. Li, K. Zhao, Y. Yu and L. Z. Zhang, Adv. Funct. Mater., 2015, 25, 2189.

34 X. C. Song, Y. F. Zheng, H. Y. Yin, J. N. Liu and X. D. Ruan, New J. Chem., 2016, 40, 130-135.

35 J. Li, L. J. Cai, J. Shang, Y. Yu and L. Z. Zhang, Adv. Mater., 2016, 28, 4059-4064.

36 X. M. Jia, J. Cao, H. L. Lin, M. Y. Zhang, X. M. Guo and S. F. Chen, RSC Adv., 2016, 6, 55755-55763.

37 Q. Y. Wang, R. C. Jin, M. Zhang and S. M. Gao, J. Alloys Compd., 2017, 690, 139-144.

38 X. D. Chen, X. Su, H. M. Duan, B. L. Liang, Y. Q. Huang and T. Zhang, Catal. Today, 2017, 281, 312-318. 
39 C. Regmi, Y. K. Kshetri, S. K. Ray, R. P. Pandey and S. W. Lee, Appl. Surf. Sci., 2017, 392, 61-70.

40 W. W. Wu, D. Q. Chen, Y. Zhou, Z. Y. Wan and Z. G. Ji, J. Alloys Compd., 2016, 682, 275-283.

41 J. X. Xia, M. X. Ji, W. B. Li, J. Di, H. Xu, M. Q. He, Q. Zhang and H. M. Li, Colloids Surf., A, 2016, 489, 343-350.

42 Z. J. Zhang, W. Z. Wang and Y. Zhou, Appl. Surf. Sci., 2014, 319, 250-255.

43 X. F. Chang, M. A. Gondal, B. Zhang, Y. S. Liu, G. B. Ji and X. M. Zhang, Appl. Surf. Sci., 2012, 258, 7826-7832.

44 C. Lai, M. M. Wang, G. M. Zeng, Y. G. Liu, D. L. Huang, C. Zhang, R. Z. Wang, P. Xu, M. Cheng, C. Huang, H. P. Wu and L. Qin, Appl. Surf. Sci., 2016, 390, 368-376.

45 X. Pan, Y. Zhao, S. Liu, C. L. Korzeniewski, S. Wang and Z. Y. Fan, ACS Appl. Mater. Interfaces, 2012, 4, 3944-3950.

46 E. Yazici, S. Yanik and M. B. Yilmaz, Carbon, 2017, 111, 822827.

47 T. T. Jiang, J. L. Li, Z. Sun, X. J. Liu, T. Lu and L. K. Pan, Ceram. Int., 2016, 42, 16463-16468.

48 S. Y. Song, W. Gao, X. Wang, X. Y. Li, D. P. Liu, Y. Xing and H. J. Zhang, Dalton Trans., 2012, 41, 10472-10476.

49 H. P. Li, T. X. Hu, N. Du, R. J. Zhang, J. Q. Liu and W. G. Hou, Appl. Catal., B, 2016, 187, 342-349.

50 H. Li, J. Shang, Z. H. Ai and L. Z. Zhang, J. Am. Chem. Soc., 2015, 137, 6393-6399.

51 Y. J. Mai, D. Zhang, Y. Q. Qiao, C. D. Gu, X. L. Wang and J. P. Tu, J. Power Sources, 2012, 216, 201-207.
52 Z. Qiong, H. Y. Qiu, C. X. Gang, H. D. Hu, L. L. Jiang, Y. Ting and J. L. Li, Chin. Sci. Bull., 2011, 56, 331-339.

53 Z. S. Liu, Z. L. Liu, J. L. Liu, J. W. Zhang, T. F. Zhou and X. Ji, Mater. Res. Bull., 2016, 76, 256-263.

54 Z. H. Ai, W. K. Ho and S. C. Lee, J. Phys. Chem. C, 2011, 115, 25330-25337.

55 D. Wu, B. Wang, W. Wang, T. C. An, G. Y. Li, T. W. Ng, H. Y. Yip, C. M. Xiong, H. K. Lee and P. K. Wong, J. Mater. Chem. A, 2015, 3, 15148-15155.

56 M. Shang, W. Z. Wang and H. L. Xu, Cryst. Growth Des., 2009, 9, 991.

57 L. W. Zhang, T. G. Xua, X. Zhao and Y. F. Zhua, Appl. Catal., B, 2010, 98, 138.

58 H. Xu, W. Wang, W. Zhu and L. Zhou, Nanotechnology, 2006, 17, 3649.

59 G. C. Xi, K. Xiong, Q. B. Zhao, R. Zhang, H. B. Zhang and Y. T. Qian, Cryst. Growth Des., 2006, 6, 577.

60 L. S. Zhang, W. Z. Wang, Z. G. Chen, L. Zhou, H. L. Xu and W. Zhu, J. Mater. Chem., 2007, 17, 2526.

61 F. X. Wang, C. Wang, Y. J. Zhao, Z. C. Liu, Z. Chang, L. J. Fu, Y. S. Zhu, Y. P. Wu and D. Y. Zhao, Small, 2016, 12, 62076213.

62 H. Huang, X. Han, X. Li, S. Wang, P. K. Chu and Y. Zhang, ACS Appl. Mater. Interfaces, 2015, 7, 482-492.

63 X. J. Wen, C. Zhang, C. G. Niu, L. Zhang, D. W. Huang, X. Y. Wang, X. G. Zhang and G. M. Zeng, RSC Adv., 2016, 6, 4035. 